\title{
Intervenções de enfermagem para prevenção e manejo das intercorrências durante a
}

\section{diálise}

Nursing interventions for the prevention and management of intercurrences during dialysis

Intervenciones de enfermería para la prevención y gestión de intercurrencias durante la diálisis

\begin{abstract}
Resumo
Objetivo: Descrever e analisar as intervenções de enfermagem prescritas para prevenção e manejo das intercorrências durante a hemodiálise. Método: Trata-se de uma revisão integrativa da literatura, realizada entre os meses de agosto e setembro de 2021. Adotou-se a questão norteadora: "Quais as intervenções de enfermagem prescritas para prevenção e manejo das intercorrências durante a hemodiálise”. Os artigos foram coletados nas bases de dados: Literatura Latino Americana e do Caribe em Ciências da Saúde (lilacs), Base de dados em enfermagem (Bdenf), Sistema Online de Busca e Análise de Literatura Médica (Medline), via biblioteca virtual de saúde e na base de dados da Scientific Eletronic Library (Scielo). Resultados: A amostra foi composta por 10 artigos. Verificou-se que as intervenções mais frequentes foram relacionadas as orientações acerca da doença e tratamento, cuidados com o acesso, incentivo ao autocuidado, cuidado com alimentação e pratica de atividade física, manutenção do equilíbrio hídrico, prevenção de complicações como infecções, sangramentos, contaminações e obstruções do acesso. Conclusão: percebe-se que o enfermeiro tem um papel fundamental na elaboração das intervenções para prevenção e manejo das intercorrências durante a hemodiálise.
\end{abstract}

Palavras-chave: Cuidados de enfermagem; Insuficiência renal; Diálise renal.

\begin{abstract}
Objective: To describe and analyze the nursing interventions prescribed for the prevention and management of intercurrences during hemodialysis. Method: This is an integrative literature review conducted between August and September 2021. The guiding question was: "What are the nursing interventions prescribed for the prevention and management of intercurrences during hemodialysis? The articles were collected in the databases: Latin American and Caribbean Literature on Health Sciences (lilacs), Nursing Database (Bdenf), Online System for Search and Analysis of Medical Literature (Medline), via the virtual health library and the Scientific Eletronic Library (Scielo) database. Results: The sample consisted of 10 articles. It was verified that the most frequent interventions were related to orientations about the disease and treatment, care with access, incentive to self-care, care with feeding and practice of physical activity, maintenance of water balance, prevention of complications such as infections, bleeding, contaminations and access obstructions. Conclusion: it can be seen that nurses play a key role in the development of interventions for the prevention and management of intercurrences during hemodialysis.
\end{abstract}

Keywords: Nursing Care; Renal Insufficiency; Renal dialysis.

\section{Resumen}

Objetivo: Describir y analizar las intervenciones de enfermería prescritas para la prevención y manejo de complicaciones durante la hemodiálisis. Método: Se trata de una revisión integradora de la literatura, realizada entre agosto y septiembre de 2021. Se adoptó la pregunta orientadora: "Qué intervenciones de enfermería se prescriben para la prevención y manejo de las complicaciones durante la hemodiálisis". Los artículos fueron recolectados en las siguientes bases de datos: Literatura Latinoamericana y del Caribe en Ciencias de la Salud (lilas), Base de Datos en Enfermería (Bdenf), Sistema de Búsqueda y Análisis en Línea de Literatura Médica (Medline), vía biblioteca virtual de 
salud y en la base de datos deal Biblioteca Eletrónica científica (SCIELO). Resultados: La muestra estuvo conformada por 10 artículos. Se encontró que las intervenciones más frecuentes se relacionaron con guías sobre la enfermedad y tratamiento, cuidados con acceso, estímulo al autocuidado, cuidados con la alimentación y la actividad física, mantenimiento del equilibrio hídrico, prevención de complicaciones como infecciones, sangrado, contaminación y obstrucciones de acceso. Conclusión: es evidente que el enfermero tiene un papel fundamental en el desarrollo de intervenciones para la prevención y manejo de las complicaciones durante la hemodiálisis.

Palabras clave: Atención de enfermera; Insuficiencia renal; Diálisis renal.

\section{Introdução}

A insuficiência renal é definida pela perda das funções vitais dos rins, fisiologia e estrutura, e pode acontecer lentamente ou progressivamente, de forma reversível ou irreversível. Apesar de sua causa ser multifatorial, o diabetes mellitus e a hipertensão arterial tem sido os principais fatores originários da doença (Silva et al., 2017) e pode ser classificada como aguda (IRA), quando os rins perdem suas funções excretoras, reguladoras e endócrinas de maneira rápida e súbita, e crônica (IRC) quando os rins perdem suas funções de forma lenta, progressiva e irreversível ocasionando um desequilíbrio homeostático dos rins.

O tratamento pode ser realizado por meio das terapias renal substitutiva (TRS), como a hemodiálise, diálise peritoneal ou transplante renal (Brasil, 2020). Lorenzet (2018), explica que cada tratamento possui suas vantagens e desvantagens, não tendo um melhor ou pior, e sim indicações para o tratamento mais adequado conforme o perfil de cada paciente, escolha e condições clínicas. Na hemodiálise, uma máquina assume as funções dos rins, promovendo bem-estar do paciente e melhora na sua qualidade de vida e saúde, o paciente sofre com as mudanças no seu estilo de vida, atividades laborais e sociais (Brasil, 2019). Já na diálise peritoneal, uma membrana funciona como uma espécie de filtro natural através do peritônio (Lorenzet, 2018). E por fim, o transplante renal, é recebe quando o indivíduo recebe um novo rim que irá substituir o rim doente, de modo a ter de volta a homeostase do seu organismo, o que confere melhor qualidade de vida, e mais liberdade para o paciente (SBN, 2021).

Apesar do crescente avanço tecnológicos ocorrido nos últimos anos em relação ao tratamento da insuficiência renal, percebe-se que as intercorrências em especial durante a hemodiálise ainda são frequentes e motivo de grande preocupação entre os profissionais que assistem ao indivíduo. Das intercorrências mais frequentes tem-se a hipotensão arterial (Silva et al., 2018), cefaleia, mal-estar, hipertensão, hipoglicemia, câimbras, náuseas, vômito, hipervolemia, febre, calafrios, diarreia, lombalgia, dor abdominal (Evaristo et al., 2020).

Diante disto, destaca-se a importância da equipe de enfermagem que deve preparada e capacitada para prestar a assistência adequada para prevenção e manejo das intercorrências (Silva et al., 2018).

Neste aspecto, ressalta-se a importância de o enfermeiro atuar seguindo as etapas dos processos de enfermagem e, portanto, prestando uma assistência sistematizada a fim de garantir uma assistência de mais qualidade e segura ao indivíduo. Destaca-se que são cinco as etapas do processo de enfermagem (investigação, diagnóstico de enfermagem, planejamento, implementação e avaliação) (COFEN, 2009). Assim sendo, o enfermeiro quando executa suas atividades este consegue prever e antecipar as possíveis situações de risco, e, portanto, desenvolver estratégias para prevenir e manejar de maneira mais adequada as intercorrências por meio das intervenções - Nursing Interventions Classification (NIC), de modo a planejar os resultados e avaliar a resposta do indivíduo ao cuidado prestado (Silva et al., 2018).

Dessa forma, tem-se o objetivo de realizar uma busca de estudos primários na literatura que discorram sobre o tema e, portanto, descrever e analisar as intervenções de enfermagem prescritas para prevenção e manejo das intercorrências durante a hemodiálise. 


\section{Metodologia}

Trata-se de uma revisão integrativa, um método que permite a procura, avaliação crítica e a síntese das evidências, em que o produto final é o estado do conhecimento do tema investigado, a implementação de intervenções efetivas na prestação de cuidados e na redução de custos, além disso permite a identificação de fragilidades que poderão conduzir ao desenvolvimento de futuras investigações (Sousa et al., 2017).

Essa revisão foi estruturada em seis fases distintas: identificação do tema e seleção da questão de pesquisa para a elaboração da revisão integrativa; estabelecimento de critérios para inclusão e exclusão de estudos/amostragem ou pesquisa de literatura; definição das informações a serem extraídas dos estudos selecionados/categorização dos estudos; avaliação dos estudos incluídos na revisão integrativa; interpretação dos resultados e, apresentação da revisão/síntese do conhecimento (Mendes, Silveira \& Galvão, 2008).

Para responder à pergunta norteadora de estudo "Quais as intervenções de enfermagem prescritas para prevenção e manejo das intercorrências durante a hemodiálise”, utilizou-se o acrômio PICo, onde P (Problema - Prevenção e manejo das intercorrências durante a Hemodiálise); I (Interesse- intervenção de enfermagem); Co (Contexto- insuficiência renal) (Araújo, 2020).

A busca nas bases de dados foi realizada nas bases de dados da Literatura Latino-Americano em Ciências da Saúde (LILACS), Base de dados em enfermagem (BDENF), Sistema Online de Busca e Análise de Literatura Médica (MEDLINE), via biblioteca virtual de saúde e na base de dados da Scientific Eletronic Library (Scielo), entre os meses de agosto e setembro de 2021, utilizando-se os descritores controlados e não controlados, bem como MeSH, bem como os conectores booleanos AND e OR, conforme observado no Quadro 1, seguinte.

Quadro 1: Apresentação da estratégia PICo para a estratégia de busca.

\begin{tabular}{|c|c|c|c|}
\hline & $\mathbf{P}$ & $\mathbf{I}$ & Co \\
\hline Extração & $\begin{array}{l}\text { Prevenção e manejo das } \\
\text { intercorrências durante a } \\
\text { Hemodiálise }\end{array}$ & Intervenção de enfermagem & Insuficiência renal \\
\hline Conversão & Renal Dialysis & Nursing Care & Renal Insufficiency \\
\hline Combinação & $\begin{array}{c}\text { Diálise Renal } \\
\text { Diálise extracorpórea } \\
\text { hemodiálise }\end{array}$ & $\begin{array}{c}\text { Cuidados de Enfermagem Assistência de } \\
\text { Enfermagem } \\
\text { Atendimento de Enfermagem } \\
\text { Cuidado de Enfermagem } \\
\text { Gestão da Assistência de Enfermagem } \\
\text { Sistematização da Assistência de } \\
\text { Enfermagem }\end{array}$ & $\begin{array}{l}\text { Insuficiência Renal } \\
\text { Insuficiência do Rim }\end{array}$ \\
\hline construção & $\begin{array}{c}\text { (Renal Dialysis) OR } \\
\text { (Diálise Renal) OR (Diálise } \\
\text { extracorpórea) OR } \\
\text { (hemodiálise) }\end{array}$ & $\begin{array}{c}\text { (Nursing Care) OR (Cuidados de } \\
\text { Enfermagem) OR (Assistência de } \\
\text { Enfermagem) OR (Atendimento de } \\
\text { Enfermagem) OR (Cuidado de Enfermagem) } \\
\text { OR (Gestão da Assistência de Enfermagem) } \\
\text { OR (Sistematização da Assistência de } \\
\text { Enfermagem) }\end{array}$ & $\begin{array}{l}\text { (Renal Insufficiency) } \\
\text { OR (Insuficiência } \\
\text { Renal) OR } \\
\text { (Insuficiência do Rim) }\end{array}$ \\
\hline $\begin{array}{c}\text { Uso } \\
\text { LILACS } \\
\text { BDENF } \\
\text { MEDLINE }\end{array}$ & \multicolumn{3}{|c|}{$\begin{array}{l}\text { (Renal Insufficiency) OR (Insuficiência Renal) OR (Insuficiência do Rim)) AND ((Nursing Care) OR } \\
\text { (Cuidados de Enfermagem) OR (Assistência de Enfermagem) OR (Atendimento de Enfermagem) OR } \\
\text { (Cuidado de Enfermagem) OR (Gestão da Assistência de Enfermagem) OR (Sistematização da Assistência } \\
\text { de Enfermagem) ) AND ((Renal Dialysis) OR (Diálise Renal) OR (Diálise extracorpórea) OR } \\
\text { (hemodiálise)) }\end{array}$} \\
\hline SCIELO & \multicolumn{3}{|c|}{$\begin{array}{l}\text { ((Renal Insufficiency) OR (Insuficiência Renal) OR (Insuficiência do Rim)) AND ((Nursing Care) OR } \\
\text { (Cuidados de Enfermagem) OR (Assistência de Enfermagem) OR (Atendimento de Enfermagem) OR } \\
\text { (Cuidado de Enfermagem) OR (Gestão da Assistência de Enfermagem) OR (Sistematização da Assistência } \\
\text { de Enfermagem)) AND ((Renal Dialysis) OR (Diálise Renal) OR (Diálise extracorpórea) OR } \\
\text { (hemodiálise)) }\end{array}$} \\
\hline
\end{tabular}


Foram incluídos artigos originais que respondiam à pergunta da pesquisa e excluídos editoriais, teses, dissertações, monografias, manuais, estudos duplicados, estudos de revisão da literatura. Inicialmente foram resgatados 937 estudos, sendo 550 na MEDLINE, 206 na BDENF, 198 na LILACS e 147 na Scielo. Após uso dos critérios de inclusão e inclusão restaram 438. Após a leitura dos títulos e resumos, obteve-se 10 para a amostra final.

A coleta de dados foi realizada com auxílio de um formulário com perguntas semiestruturadas elaborado pelos pesquisadores e subdividido em duas partes a fim de facilitar a coleta, organização e disposição dos dados, sendo a Parte I Perfil das produções (Título, ano de publicação, autores, idioma, abordagem e a base de dados) e Parte II - Resultados em evidência (intervenção de enfermagem para prevenção e manejo das intercorrências). Os dados coletados foram organizados em um quadro do Microsoft Office Excel e descritos em quadros. Foi realizado uma análise estatística descritiva simples.

\section{Resultados}

Inicialmente foram identificados 937 estudos, após aplicação dos critérios de exclusão e inclusão, obteve-se 18 artigos, no qual 5 não condiziam com o objetivo proposto e 3 apresentaram duplicidade, portanto 10 artigos compuseram a amostra final.

$\mathrm{Na}$ Tabela 1 tem-se a distribuição das produções cientificas de acordo com ano de publicação, periódico de publicação e abordagem metodológica. Nota-se que o maior número de publicações ocorreu entre os anos de 2017 e 2018 (60\%), na Revista de Enfermagem da UFPE On Line (20\%).

Tabela 1: Distribuição das produções cientificas de acordo com ano de publicação, periódico de publicação e abordagem metodológica $(\mathrm{n}=10)$. Teresina, PI, 2021.

$\begin{array}{lcc}\text { Ano de publicação } & \mathbf{N}^{\circ} & \% \\ 2017-2018 & 6 & 60 \\ 2019-2020 & 3 & 30 \\ 2021 & 1 & 10 \\ \quad & & \\ \text { Periódicos } \quad 1 & 10 \\ \quad \text { Acta Paulista de Enfermagem } & 1 & 10 \\ \text { Rev. Gaúcha de Enfer. } & 1 & 10 \\ \text { Enfermagem Global } & 2 & 20 \\ \text { Rev. de Enfer. UFPE On Line } & 1 & 10 \\ \text { Rev. de Enfer. Do Cento-Oeste Mineiro } & 1 & 10 \\ \text { Texto e Contexto Enfer. } & 1 & 10 \\ \text { Esc Anna Nery } & 1 & 10 \\ \text { Irish Journal of Medical Science } & 1 & 10 \\ \text { Rev. Latino-Americana de Enfer. } & & \\ & & 80 \\ \text { Abordagem metodológica } & 8 & 20 \\ \text { Quantitativo } & 2 & \\ \text { Qualitativo } & & \end{array}$

Fonte: Autores.

No Quadro 2 tem-se a apresentação dos estudos, conforme o título e objetivo da pesquisa. 
Quadro 2. Apresentação dos estudos, conforme o título e objetivo da pesquisa

\begin{tabular}{|c|c|c|}
\hline $\mathbf{n}$ & Titulo & Objetivo \\
\hline 1 & $\begin{array}{l}\text { Pacientes em diálise peritoneal: } \\
\text { associação entre diagnósticos de } \\
\text { enfermagem e seus componentes }\end{array}$ & $\begin{array}{c}\text { Identificar nos pacientes, os diagnósticos, características } \\
\text { definidoras e fatores relacionados }\end{array}$ \\
\hline 2 & $\begin{array}{c}\text { Validação de intervenções e atividades } \\
\text { de enfermagem para pacientes em } \\
\text { terapia hemodialítica }\end{array}$ & $\begin{array}{c}\text { Relacionar as intervenções prioritárias e complementares e } \\
\text { suas atividades de enfermagem }\end{array}$ \\
\hline 3 & $\begin{array}{l}\text { Diagnósticos e intervenções de } \\
\text { enfermagem no domínio da segurança } \\
\text { e proteção do paciente em hemodiálise }\end{array}$ & $\begin{array}{l}\text { Discutir os diagnósticos e as possíveis intervenções de } \\
\text { enfermagem e suas atividades }\end{array}$ \\
\hline 4 & $\begin{array}{l}\text { Ensaio clínico para o controle da } \\
\text { ingestão hídrica de pacientes em } \\
\text { tratamento hemodialítico }\end{array}$ & $\begin{array}{c}\text { Analisar as respostas dos pacientes após a aplicação de } \\
\text { intervenção educativa e motivacional }\end{array}$ \\
\hline 5 & $\begin{array}{l}\text { Intradialytic Hypotension prevalence, } \\
\text { influencing factors, and Nursing } \\
\text { interventions: prospective results of } \\
744 \text { hemodialysis sessions }\end{array}$ & $\begin{array}{c}\text { Avaliar a presença de hipotensão intradialitica e as } \\
\text { intervenções de enfermagem }\end{array}$ \\
\hline 6 & $\begin{array}{l}\text { Diagnóstico, resultado e intervenção de } \\
\text { enfermagem no paciente com cateter } \\
\text { para hemodiálise }\end{array}$ & $\begin{array}{l}\text { Estabelecer relação entre os diagnósticos, resultados e } \\
\text { intervenções no paciente renal crônico }\end{array}$ \\
\hline 7 & $\begin{array}{c}\text { Nursing interventions for } \\
\text { complications presented during } \\
\text { hemodialysis in critically ill } \\
\text { Patients }\end{array}$ & $\begin{array}{c}\text { Verificar quais são as complicações e as intervenções } \\
\text { durante a hemodiálise }\end{array}$ \\
\hline 8 & $\begin{array}{c}\text { Contextos de experiência de estar } \\
\text { (des)confortável de pacientes com } \\
\text { doença renal crônica }\end{array}$ & $\begin{array}{l}\text { Analisar as experiências de conforto e desconforto nos } \\
\text { pacientes em hemodiálise }\end{array}$ \\
\hline 9 & $\begin{array}{c}\text { Efeitos de uma intervenção } \\
\text { psicoterapêutica breve de apoio em } \\
\text { pacientes quase-experimental }\end{array}$ & $\begin{array}{l}\text { Avaliar o paciente antes e depois da aplicação de uma } \\
\text { intervenção psicoterapêutica }\end{array}$ \\
\hline 10 & $\begin{array}{c}\text { Intervenções de enfermagem no } \\
\text { paciente em hemodiálise por cateter } \\
\text { venoso central }\end{array}$ & $\begin{array}{l}\text { Discutir as intervenções feitas por enfermeiros em pacientes } \\
\text { com cateter venoso central }\end{array}$ \\
\hline
\end{tabular}

Fonte: Autores.

No Quadro 3 tem-se as principais intercorrências durante a dialise e as respectivas intervenções de enfermagem prescritas para prevenir e manejar estas intercorrências. 
Quadro 3: Quadro com as principais intercorrências e as respectivas intervenções para prevenção e manejo das mesmas.

\begin{tabular}{|c|c|c|}
\hline $\mathbf{n}^{\circ}$ & $\begin{array}{l}\text { Principais intercorrências durante a } \\
\text { dialise }\end{array}$ & $\begin{array}{c}\text { Intervenções de enfermagem para prevenção e manejo } \\
\text { das intercorrências durante a diálise }\end{array}$ \\
\hline 01 & 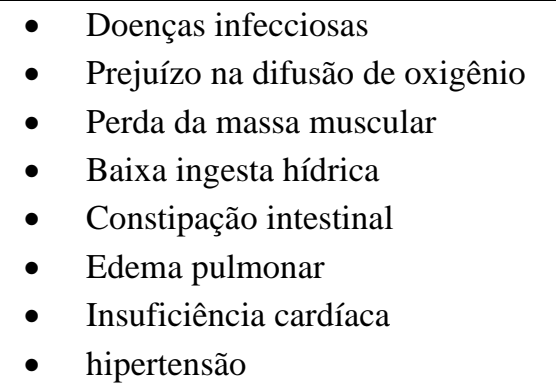 & $\begin{array}{l}\text { - Orientação sobre ações que reduzem os riscos } \\
\text { identificados, alimentação, exercícios físicos } \\
\text { - } \text { Observar sinais e sintomas } \\
\text { - } \text { Higienizar as mãos ao ter contato com o paciente } \\
\text { - } \text { Explicar a causa da fadiga } \\
\text { - } \text { Fazer o controle de energia entre perdas e consumo } \\
\text { - Controle hídrico, controle hidroeletrolítico e } \\
\text { monitoração hídrica }\end{array}$ \\
\hline 02 & $\begin{array}{ll}\text { - } & \text { Aumento de peso } \\
\text { - } & \text { Alterações da pressão arterial } \\
\text { - } & \text { Edema } \\
\text { - } & \text { Distensão de veia jugular } \\
\text { - } & \text { Dispneia } \\
\text { - } & \text { Congestão pulmonar } \\
\text { - } & \text { Derrame pleural } \\
\text { - } & \text { Eletrolíticos alterados } \\
\text { - } & \text { Alterações na urina } \\
\text { - } & \text { Ansiedade } \\
\text { - } & \text { Mudança no estado mental } \\
\end{array}$ & $\begin{array}{l}\text { - } \quad \text { Manter o equilíbrio hídrico do paciente } \\
\text { - } \\
\text { - } \\
\text { Apoio emocional } \\
\text { Redão da ansiedade }\end{array}$ \\
\hline 03 & $\begin{array}{ll}\text { - } & \text { Infecções } \\
\text { - } & \text { Hipotermia } \\
\text { - } & \text { Sangramentos }\end{array}$ & $\begin{array}{l}\text { - Controlar as infecções } \\
\text { - Prevenir sangramento devido a uremia em pacientes } \\
\text { com DRC e o uso de anticoagulantes na hemodiálise } \\
\text { - Controlar anafilaxia ao identificar o fator de risco } \\
\text { que seria a exposição a alérgenos Regulação de } \\
\text { temperatura. }\end{array}$ \\
\hline 04 & $\begin{array}{l}\text { - } \quad \text { Alta ingestão hídrica } \\
\text { - } \quad \text { Ansiedade }\end{array}$ & $\begin{array}{ll}\text { - } & \text { Orientação quanto ao autocuidado } \\
\text { - } & \text { Orientar quanto o controle da ingestão de líquidos } \\
\text { - } & \text { Realizar atividade educativa e motivacional. }\end{array}$ \\
\hline 05 & $\begin{array}{ll}\text { - } & \text { Hipotensão } \\
\text { - } & \text { Fadiga } \\
\text { - } & \text { Câimbras } \\
\text { - } & \text { Sudorese intensa }\end{array}$ & $\begin{array}{ll}\text { - } & \text { Interromper a ultrafiltração } \\
\text { - } & \text { Administrar solução salina } \\
\text { - } & \text { Colocar o paciente em posição de trendelenburg. }\end{array}$ \\
\hline 06 & $\begin{array}{ll}\text { - } & \text { Doenças infecciosas } \\
\text { - } & \text { Edema } \\
\text { - } & \text { Obstrução do lúmen } \\
\end{array}$ & $\begin{array}{l}\text { - Manter os cuidados com a manutenção do acesso } \\
\text { vascular }\end{array}$ \\
\hline 07 & $\begin{array}{ll}\text { - } & \text { Hipotensão } \\
\text { - } & \text { Arritmias } \\
\text { - } & \text { Hipoglicemia } \\
\text { - } & \text { Coagulação do circuito } \\
& \text { extracorpóreo } \\
\text { - } & \text { Hipotermia } \\
\end{array}$ & $\begin{array}{l}\text { - } \text { Controlar e tratamento da hipoglicemia } \\
\text { - } \quad \text { Realizar a monitoração hídrica } \\
\text { - } \quad \text { Realizar a regulação hemodinâmica } \\
\text { - } \quad \text { Manter os cuidados cardíacos } \\
\text { - } \quad \text { Manter o acesso vascular central/dialise. }\end{array}$ \\
\hline 08 & $\begin{array}{l}\text { - } \quad \text { Mobilidade prejudicada } \\
\text { - } \quad \text { Ansiedade }\end{array}$ & $\begin{array}{ll}\text { - } & \text { Manter as medidas de conforto } \\
\text { - } & \text { Realizar atividade educativa e de apoio }\end{array}$ \\
\hline
\end{tabular}




\begin{tabular}{|c|c|c|}
\hline & $\begin{array}{ll}\text { - } & \text { Infecção } \\
\text { - } & \text { Sangramentos } \\
\text { - } & \text { Alergia } \\
\text { - } & \text { Contaminação }\end{array}$ & \\
\hline 09 & - $\quad$ Ansiedade & $\begin{array}{l}\text { - Entrevista para buscar dados de pacientes sobre o } \\
\text { conforto e desconforto que estejam sofrendo } \\
\text { - Realizar técnica de relaxamento com imagens, } \\
\text { musicas } \\
\text { - Orientações verbais }\end{array}$ \\
\hline 10 & $\begin{array}{ll}\text { - } & \text { Infecção } \\
\text { - } & \text { Alergia } \\
\text { - } & \text { Contaminação } \\
\text { - } & \text { Variação de temperatura }\end{array}$ & $\begin{array}{l}\text { - } \text { Aferir a temperatura no pré e pós tratamento } \\
\text { - } \quad \text { Manter o curativo oclusivo } \\
\text { - } \text { Monitorar os sinais e sintomas } \\
\text { - } \text { Manter técnica asséptica na realização do curativo } \\
\text { - } \quad \text { Trocar as tampas dos protetores após cada sessão de } \\
\text { dialise } \\
\text { - } \text { Manter a permeabilidade do cateter } \\
\text { Orientar o paciente e família sobre a manutenção do } \\
\text { cateter. }\end{array}$ \\
\hline
\end{tabular}

Fonte: Autores

No Gráfico 1, tem-se os principais diagnósticos de enfermagem elaborados após a análise dos dados coletados em cada artigo.

Gráfico 1: Diagnósticos de enfermagem.

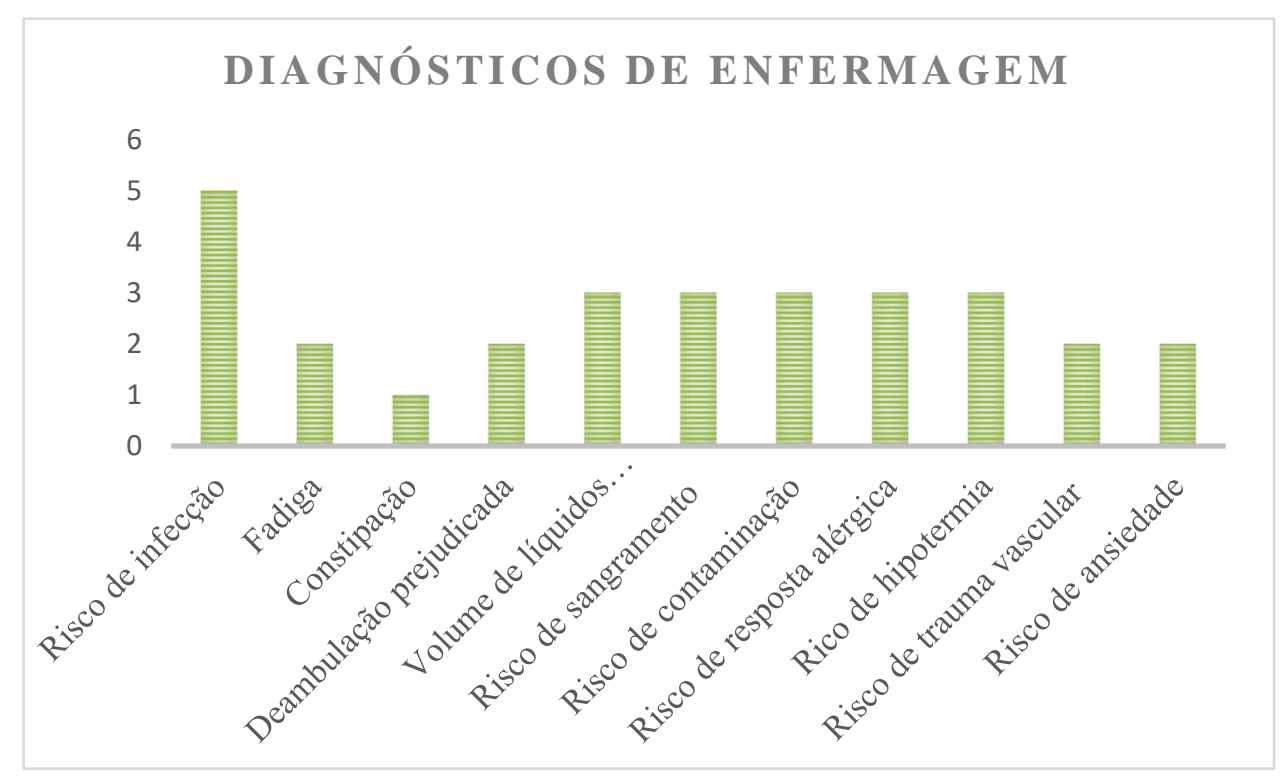

Fonte: Autores.

\section{Discussão}

A equipe de enfermagem é o grupo que mais colabora na atuação do tratamento de diálise, a observação continuada durante os procedimentos é de grande importância pois viabiliza um diagnóstico precoce sobre as intercorrências e a equipe consegue estar sempre alerta para intervir imediatamente frente a situação de emergência, em vista disso, ocasiona na diminuição 
da possibilidade de agravo da situação clínica do paciente e redução do risco de mortalidade (Evaristo et al., 2020).

Com base nos estudos de Campos et al. (2019) foi evidenciado os principais diagnósticos de enfermagem nos pacientes em tratamento para ressaltar as intervenções necessárias para cada diagnóstico encontrado. Os autores frisam que os profissionais devem ser altamente capacitados e estarem em busca de aprimoramento de suas habilidades, explicam que a equipe deve orientar sobre as ações necessárias para o autocuidado evitando possíveis infecções, fadiga, deambulação prejudicada, constipação e volumes de líquidos excessivos. Além disso, importante analisar as condições de moradia dos pacientes pois afeta diretamente no desempenho do autocuidado.

Lucena et al. (2017) aborda em sua pesquisa intervenções de enfermagem a serem aplicadas nos pacientes em terapia hemodialítica e as atividades propostas foram classificadas em prioritárias, complementares e intervenções não essenciais. O resultado do estudo indicou a intervenção controle hídrico como prioritária na assistência de enfermagem, dessa maneira, é apresentado um quadro com as atividades para serem executadas com intuito de obter esse controle hídrico. $\mathrm{O}$ cuidado da equipe a esses pacientes são de monitorar os sinais vitais, o equilíbrio hídrico, a variação de peso, a reação do paciente sobre o tratamento, sua compreensão, alimentos e líquidos ingeridos e condição nutricional, é também importante a orientação e auxílio aos pacientes e familiares sobre como realizar o autocuidado para evitar possíveis intercorrências e explicar as limitações.

Em seu estudo Aguiar e Guedes (2017) analisaram diagnósticos que foram identificados a partir de dados coletados, sendo eles, risco de infecção, risco de sangramento, risco de contaminação, risco de resposta alérgica e risco de hipotermia. Ao detectar esses diagnósticos foi estabelecido intervenções para a prevenção destas possíveis complicações sendo eles controlem de infecção, precauções contra sangramento, terapia de hemodiálise, controle de anafilaxia e regulação de temperatura. Demonstram no estudo que a equipe é fundamental no desenvolvimento de ações preventivas e educacionais por todos os diagnósticos serem fatores de risco e a importância do profissional se atentar aos detalhes pois podem influenciar no desenvolvimento do tratamento.

Oller et al. (2018) estudaram a aplicação de intervenção educativa e motivacional para o controle do consumo de liquido com 192 participantes. O estudo foi feito de acordo com a Teoria Social Cognitiva de Bandura, utilizando uma intervenção em forma de vídeo a fim de educar sobre a ingestão de liquido e orientar sobre o autocuidado para evitar intercorrências. É evidenciado no estudo a necessidade por parte dos profissionais, a constante busca por aprimoramento de suas habilidades e obtenção de conhecimentos atualizados.

Em um estudo que foi realizado por meio de uma análise de 744 sessões de hemodiálise com 124 pacientes em um centro de diálise na Turquia, concluiu que a hipotensão é uma intercorrência bastante comum durante as sessões de hemodiálise, onde dos 124 pacientes que participaram do estudo 51,6\% dos indivíduos apresentaram hipotensão durante a sessão. Os mesmos apresentaram sintomas comuns de fadiga, câimbras e sudorese intensa. Afim de solucionar tal intercorrência e diminuir os riscos de agravamento do quadro do paciente, as principais intervenções de enfermagem foram: interromper a ultrafiltração, colocar os pacientes em posição de trendelenburg e administrar solução salina isotônica. Segundo Ozen e Cepken (2020) tais intervenções são vitais pois impossibilitou danos graves aos indivíduos, como também o aumento da morbidade cardiovascular entre eles. Tendo em vista que os sintomas diminuem a qualidade de vida dos pacientes, tais intervenções se aplicadas de imediato evita tais danos e até mais graves a saúde dos clientes.

Com base na sua pesquisa, Guimarães et al. (2017) observaram que o uso do cateter venoso central traz riscos de infecção para os pacientes, portando foi estabelecido as intervenções manutenção do acesso e cuidado com dispositivo vascular para evitar risco de trauma vascular e risco de infecção. $O$ estudo expõe que o enfermeiro tem ações preventivas e educacionais quanto ao dispositivo, é imprescindível as orientações acerca do acesso, em como devem cuidar e evitar situações que possam causar riscos.

Em outra amostra analisada, intitulada como: "Nursing interventions for complications presented during hemodialysis 
in critically ill patients. Silva et al. (2018) analisaram as principais intercorrências durante o tratamento, após identificadas, elaboraram intervenções de enfermagem para evitar essas complicações. As intervenções seriam manutenção do acesso com atividades de monitorar sinais e sintomas, monitorar o local de acesso e heparinizar o cateter, foi também indicado a ação de controle de arritmias, regulação hemodinâmica através da ausculta cardíaca, manter o equilíbrio hídrico, monitorização hídrica com atividades de monitorização de sinais vitais, valores eletrolíticos, analise da urina, verificar variação de peso, verificar presença de edema, administrar líquidos ou restringi-los, há também, controle de hipoglicemia identificando riscos, sinais e sintomas de hipoglicemia e tratamento da hipotermia. É preciso que o profissional tenha conhecimento para detectar essas complicações.

Dentre as intervenções de enfermagem no contexto saúde-doença, tem-se a de proporcionar ao paciente um ambiente confortável. De acordo com uma pesquisa realizada em uma clínica de diálise no Ceará com pacientes renais crônicos cujo objetivo consistiu em estabelecer os contextos da experiência de estar (des)confortável, conforme percepções de pacientes com a doença, durante a hemodiálise. Dentre os aspectos analisados, tem-se os socioculturais em que os pacientes entrevistados relataram uma busca incessante pela integridade do conforto social. Segundo Santiago et al. (2020), a equipe de enfermagem deve proporcionar intervenções que promovam o conforto nesse domínio, no qual consiste na promoção das relações interpessoais e familiares, intervenções educacionais pois as mesmas tem papel primordial nesse contexto, pois tais intervenções proporcionam conforto e alivio aos pacientes.

Manzini et al (2021) tiveram o objetivo de analisar os resultados de uma intervenção psicoterapêutica, envolvendo 17 participantes em 2 unidades de dialise. Utilizaram nesse estudo a intervenção Relaxamento, Imagens Mentais e Espirituais (RIME), sua técnica busca relaxamento do paciente, imaginação dirigida e elementos da espiritualidade. Teve o objetivo de contribuir com o fortalecimento espiritual e aumentar a resiliência dos pacientes frente as dificuldades do tratamento.

Por fim, Guimarães et al. (2017) buscaram discutir as intervenções de enfermagem prescritas aos pacientes em hemodiálise, exemplificando as ações e como realiza-las. Foi selecionada 2 intervenções: cuidado com o dispositivo vascular e manutenção do acesso para diálise, por haver risco de infecção é preciso que a equipe cuide do acesso evitando assim contaminação e devem orientar como proceder o autocuidado aos pacientes. No estudo ainda foram formuladas também 8 atividades para a equipe proceder: aferir a temperatura antes de pois do tratamento, cuidar do curativo, verificar se a presença de sinais e sintomas que possam ligar a infecção, realizar procedimentos com técnica asséptica, manter precaução padrão, prevenir o acesso de obstrução e infecção, trocar as trompas protetoras e realizar um processo educacional aos pacientes e familiares sobre o autocuidado.

\section{Conclusão}

Conclui-se que é fundamental o enfermeiro prestar a assistência embasado no processo de enfermagem, assim, destacase que a elaboração das intervenções de enfermagem é fundamental para a prevenção e manejo das possíveis intercorrências durante a diálise, de modo a proporcionar maior conforto, reduzir os agravamentos e a morbimortalidade relacionada ao processo de adoecimento.

Para isto é primordial que os profissionais enfermeiro e demais profissionais da equipe interdisciplinar tenham capacidade e habilidade para atender as necessidades dos indivíduos durante o tratamento e, portanto, proporcionar melhor qualidade de vida ao indivíduo e reduzir as possíveis complicações do tratamento. Em vista disso se faz necessário um maior aprofundamento de novos estudos com está temática, afim de propor aos pacientes dialíticos uma assistência mais adequada durante as intercorrências. 
Research, Society and Development, v. 10, n. 15, e206101522980, 2021

(CC BY 4.0) | ISSN 2525-3409 | DOI: http://dx.doi.org/10.33448/rsd-v10i15.22980

\section{Referências}

Araújo, W. C. O. (2020). Recuperação da informação em saúde: construção, modelos e estratégias. Convergências em Ciência da Informação. 3(2), 100-34, maio/ago. http://www.repositorio.ufc.br/handle/riufc/52993.

Brasil. (2020). Dia mundial do rim. Ministério da Saúde. Biblioteca Virtual em Saúde. http://bvs.saude.gov.br/component/content/article?id=3138

Brasil. (2019). Hemodiálise. Ministério da Saúde. Biblioteca Virtual em Saúde. http://bvsms.saude.gov.br/dicas-em-saude/2988-hemodialise

Campos, M. X. B., Dutra, E. J. O., Silva, C. J. A., Menezes, H. F., Santos, R. S. C., \& Silva, R. A. R. (2019). Pacientes em diálise peritoneal: associação entre diagnósticos de enfermagem e seus componentes. Acta Paulista de Enfermagem, 32(6), 651-8. https://www.scielo.br/j/ape/a/KVdYHdQjGX7ypNMqHSmB6Fq/?lang=pt

Conselho federal de enfermagem (COFEN). (2009). Resolução COFEN $n^{o}$ 358/2009, de 15 de outubro de 2009. http://www.cofen.gov.br/resoluo-cofen3582009_4384.html.

Evaristo, L. S., Cunha, A. P., Morais, C. G., Samselski, B. J. L., Esposito, E. P., Miranda, M. K. V., \& Gouvea-e-Silva, L. F. (2020). Complicações durante a sessão de hemodiálise. Avances en Enfermería. 38(3), 316-24. DOI: 10.15446/av.enferm.v38n3.84229.

Freire, S. M. L., Melo, G. A. A., Lima, M. M. S., Silva, R. A., Caetano, J. Á., \& Santiago, J. C. S. (2020). Contextos de experiência de estar (des)confortável de pacientes com doença renal crônica. Escola Anna Nery. 24(4). <https://doi.org/10.1590/2177-9465-EAN-2019-0326>

Guimarães, G. L., Goveia, V. R., Mendonza, I. Y. Q., Corrêa, A. R., Matos, S. S., \& Guimarães, J. O. (2017). Intervenções de enfermagem no paciente em hemodiálise por cateter venoso central. Rev enferm UFPE. 11(3):1127-35, https://periodicos.ufpe.br/revistas/revistaenfermagem/article/view/13487/16205.

Guimarães, G. L., Mendoza, I. Y. Q., Werli-Alvarenga, A., Barbosa, J. A. G., Corrêa, A. R., Guimarães, J. O., Guimarães, M. O., \& Chianca, T. C. M. (2017) Diagnóstico, resultado e intervenção de enfermagem no paciente com cateter para hemodiálise. J. Nurs. UFPE, 11(11):4334-42, https://periodicos.ufpe.br/revistas/revistaenfermagem/article/view/23544/24933.

Lima-Aguiar, L., \& Cavalcante-Guedes, M.V. (2017). Diagnósticos e intervenciones de enfermería del dominio seguridad y protección de los pacientes en hemodiálisis. Enfermaría global. Murcia. 16(47), 1-37. https://dx.doi.org/10.6018/eglobal.16.3.248291.

Lorenzet, M. (2018). Perfil de pacientes em terapia renal substitutiva em um serviço especializado. UFFS. https://rd.uffs.edu.br/handle/prefix/3159.

Lucena, A. F., Magro, C. Z., Proença, M. C. C., Pires, A. U. B., Moraes, V. M., \& Aliti, G. B. (2017). Validação de intervenções e atividades de enfermagem para pacientes em terapia hemodialítica. Revista Gaúcha de Enfermagem. 38(3), e66789. <https://doi.org/10.1590/1983-1447.2017.03.66789>.

Manzini, C. S. S., Damasceno, V. A. M., Santos, D. G. M., Neto, M. M., Sousa, C. N., Elias, A. C. A., \& Orlandi, F. S. (2021). Efeitos de uma intervenção psicoterapêutica breve de apoio em pacientes hemodialisados: estudo quase-experimental. Texto Contexto Enferm [Internet]. https://doi.org/10.1590/1980-265XTCE-2020-0116.

Mendes, K. D. S., Silveira, R. C. C. P., \& Galvão, C. M. (2008). Revisão integrativa: método de pesquisa para a incorporação de evidências na saúde e na enfermagem. Texto \& contexto enfermagem, 17(4), 758-64. https://www.scielo.br/j/tce/a/XzFkq6tjWs4wHNqNjKJLkXQ/?lang=pt.

Oller, G. A. S. A. O., Oliveira, M. P., Cesarino, C. B., Teixeira, C. R. S., Costa, J. A. C., \& Kusumota, L. (2018). Clinical trial for the control of water intake of patients undergoing hemodialysis treatment. Rev. 26:e3091. https://www.scielo.br/j/rlae/a/CZgsMMmsMnhrW94CqzyWsyS/?lang=pt\&format=pdf

Ozen, N., \& Cepken, T. (2020). Intradialytic hypotension prevalence, influencing factors, and nursing interventions: prospective results of 744 hemodialysis sessions. Ir J Med Sci. 189(4):1471-6. DOI: https://doi.org/10.1007/s11845-020-02249-9.

Silva, A. A., Pires, F. L., Pereira, K. C., Andrade, L. C. V., Leite, L. M., Góes, M. A., Oliveira, V. F. S., Gonzaga, M. F. N., Guidi, L. R., \& Pompeu, L. C. (2017). O processo de enfermagem (PE) - Sistematização da assistência de enfermagem (SAE) no paciente com insuficiência renal. Revista Saúde em Foco. https://portal.unisepe.com.br/unifia/wp-content/uploads/sites/10001/2018/06/073_processo_enfermage_insuficiencia_renal.pdf.

Silva, A. F. S., Magalhães, D. M., Rocha, P. R. S., \& Silva, R. F. (2018). Intervenções de Enfermagem Para Complicações Apresentadas Durante a Hemodiálise em Pacientes Críticos. Revista de Enfermagem do Centro-Oeste Mineiro. 8:e2327. http://seer.ufsj.edu.br/index.php/recom/article/view/2327/1863.

Sousa, L. M. M., Marques-Vieira, C., Severino, S., \& Antunes, V. (2017). Metodologia de Revisão Integrativa da Literatura em Enfermagem. Revista Investigação Enfermagem. $17-26$. https://www.researchgate.net/publication/321319742_Metodologia_de_Revisao_Integrativa_da_Literatura_em_Enfermagem.

Sociedade Brasileira De Nefrologia. (2021). Hemodiálise. https://www.sbn.org.br/. 